\title{
Dramatic Enhancement of Tip-Enhanced Raman Scattering Mediated by Atomic Point Contact Formation
}

\author{
Shuyi Liu, Borja Cirera, Yang Sun, Ikutaro Hamada, Melanie Müller, Adnan Hammud, Martin Wolf, \\ and Takashi Kumagai*
}

Cite This: Nano Lett. 2020, 20, 5879-5884

Read Online

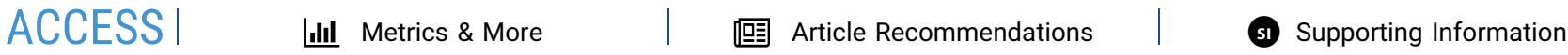

ABSTRACT: Tip-enhanced Raman scattering (TERS) in ångström-scale plasmonic cavities has drawn increasing attention. However, Raman scattering at vanishing cavity distances remains unexplored. Here, we show the evolution of TERS in transition from the tunneling regime to atomic point contact (APC). A stable $\mathrm{APC}$ is reversibly formed in the junction between an Ag tip and ultrathin $\mathrm{ZnO}$ or $\mathrm{NaCl}$ films on the $\mathrm{Ag}(111)$ surface at $10 \mathrm{~K}$. An abrupt increase of the TERS intensity occurs upon APC formation for $\mathrm{ZnO}$, but not for $\mathrm{NaCl}$. This remarkable observation is rationalized by a difference in hybridization between the $\mathrm{Ag}$ tip and these films, which determines the contribution of charge transfer enhancement in the fused plasmonic junction. The strong hybridization between the $\mathrm{Ag}$ tip and $\mathrm{ZnO}$ is corroborated by TERS from Tunneling regime to Atomic Point Contact

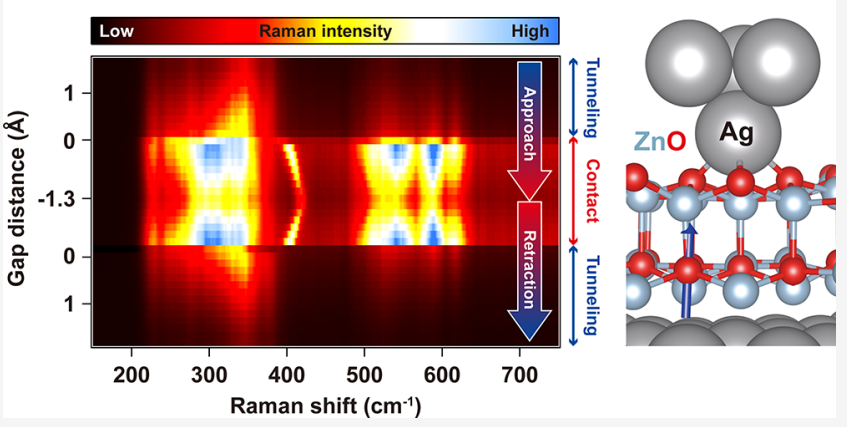
the appearance of a new vibrational mode upon APC formation, whereas it is not observed for the chemically inert $\mathrm{NaCl}$.

KEYWORDS: Tip-enhanced Raman spectroscopy, Scanning tunneling microscopy, Plamonic picocavity, Atomic point contact, Ultrathin films

$\mathrm{T}$ ERS has become a powerful tool for ultrasensitive chemical analysis in nanoscale environments. ${ }^{1}$ The ultrahigh sensitivity down to the single-molecule level relies on plasmonically enhanced Raman scattering in metallic nanocavities, so-called "hot spots". Recently, it has been demonstrated that the exceptional high sensitivity can be obtained in subnanometer plasmonic cavities (picocavities), where sharply confined electromagnetic fields generated by atomic-scale asperities play a crucial role. ${ }^{2}$ First-principle simulations have also suggested that the field confinement reaches even atomic scales, ${ }^{3,4}$ which is supported by observing characteristic cavity modes of a well-controlled plasmonic dimer in white-light scattering ${ }^{5}$ and, more recently, by remarkable ångström-resolution Raman imaging in TERS at extremely small gap distances $(<3 \AA) .{ }^{6,7}$ Therefore, elucidating the nature of plasmonic fields and light-matter interactions in atomic-scale cavities is now of key importance for both fundamental physics and practical applications.

As the cavity size approaches the ångström scale, the plasmon mode undergoes a transition from the capacitively to the conductively coupled regime. ${ }^{8}$ The plasmonic field and enhanced Raman scattering in the capacitively coupled regime can be well described by classical electromagnetics and standard Raman scattering theories. On the other hand, Raman scattering in the conductively coupled regime is governed by charge transfer plasmons and direct scattering with optically induced currents, ${ }^{9}$ where a full quantummechanical and nonequilibrium treatment becomes necessary. In addition, it was reported that qualitatively different enhanced Raman spectra are observed when a molecule is electrically fused in a plasmonic junction. ${ }^{10,11}$ Although these measurements suggest a critical impact of a chemical interaction in conductively coupled junctions, the precise control of the gap distance is lacking and the evolution of Raman scattering at vanishing distances remains elusive.

In this Letter, we reveal the evolution of TERS intensities and spectra in an atomic-scale plasmonic cavity including transition from a tunneling regime to atomic point contact (APC) formation. Using the precise control of the gap distance in low-temperature scanning tunneling microscopy (STM), a stable APC is formed in a reproducible manner at $10 \mathrm{~K}$ between an $\mathrm{Ag}$ tip and a 2-monolayer (ML) thick $\mathrm{ZnO}$ or $\mathrm{NaCl}$ film grown on an $\mathrm{Ag}(111)$ surface. The measurements of these samples with a largely different electronic structure clarify that hybridization between the sample and the Ag tip has a

Received: April 28, 2020

Revised: July 16, 2020

Published: July 17, 2020 

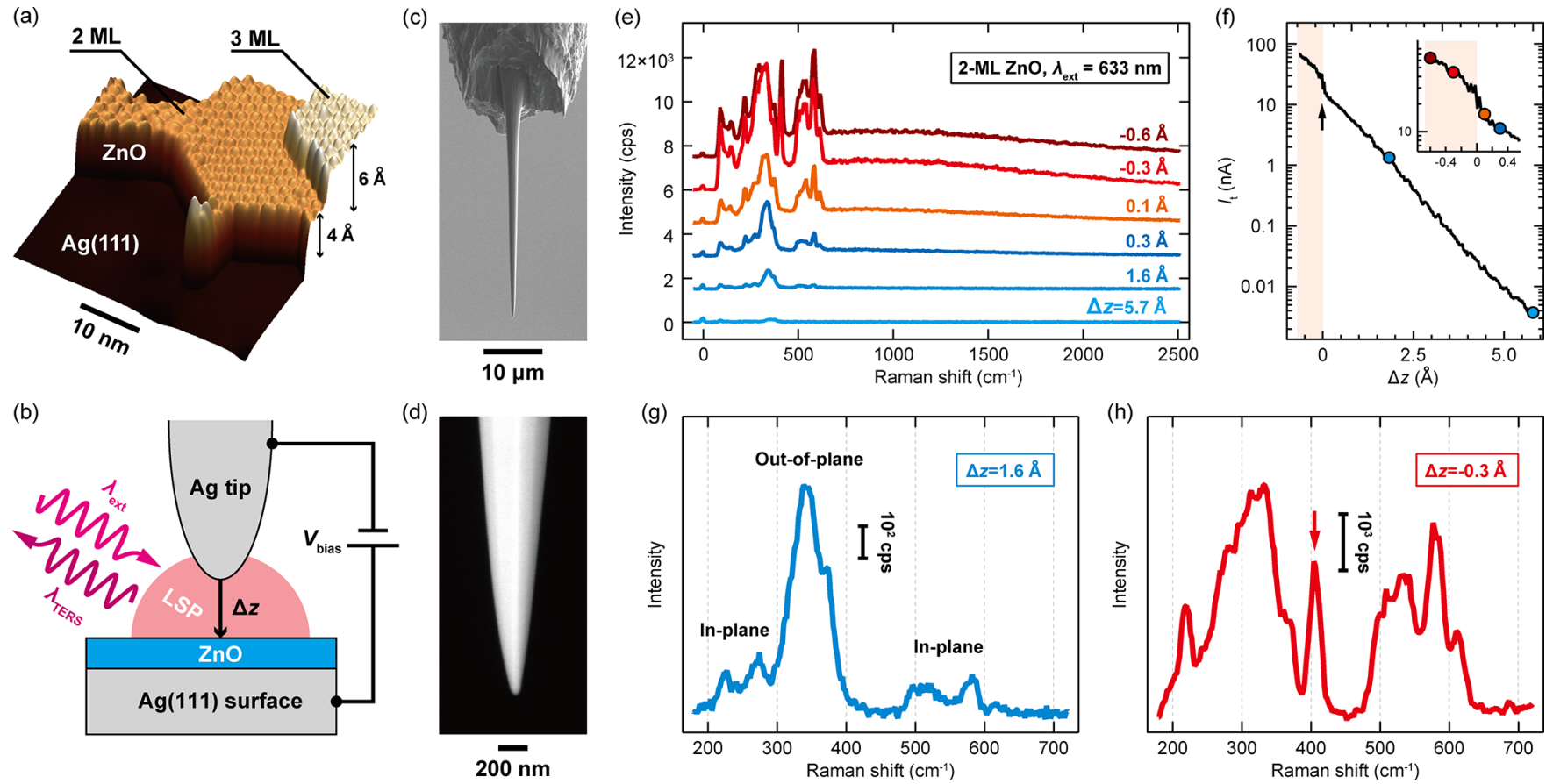

Figure 1. Gap-distance-dependent TERS measurement for 2-ML ZnO. (a) Quasi 3D STM image of ultrathin ZnO layers epitaxially grown on the $\operatorname{Ag}(111)$ surface $\left(V_{\text {bias }}=1 \mathrm{~V}, I_{\mathrm{t}}=100 \mathrm{pA}\right)$. (b) Schematic of the measurement configuration. (c-d) Scanning electron micrograph of the FIB Ag tip. (e) TER spectra of 2-ML $\mathrm{ZnO}$ recorded at different $\Delta z$ as indicated in the figure (Ag tip, $\lambda_{\text {ext }}=633 \mathrm{~nm}, F=0.29 \mathrm{~mW}_{\mu \mathrm{m}}^{-2}$, exposure time $\left(t_{\text {exp }}\right)=3 \mathrm{~s}$ per spectrum, $10 \mathrm{~K}$ ). (f) $I_{\mathrm{t}}$ versus $\Delta z$ acquired over $2-\mathrm{ML} \mathrm{ZnO}$. The shaded area indicates the contact regime. $V_{\text {bias }}$ is set to $0 \mathrm{~V}$ nominally, but there is an offset bias of $\sim 1 \mathrm{mV}$ during the measurement. $\Delta z=0$ corresponds to the distance at which point-to-contact occurs (formation of APC). The colored circles represent the point of the TER spectra in (e). The upper-right inset shows the magnified $I_{t}-\Delta z$ curve around the APC. $(\mathrm{g}-\mathrm{h})$ TER spectra in the tunneling and contact regime, respectively. The red arrow in (h) indicates the "contact mode".

critical impact on the TERS enhancement upon APC formation through the charge transfer mechanism, whereby an abrupt increase of TERS occurs in the $\mathrm{ZnO}$ film, whereas it is absent for the chemically inert $\mathrm{NaCl}$ films.

Figure 1a displays an STM image of ultrathin $\mathrm{ZnO}$ films epitaxially grown on the $\operatorname{Ag}(111)$ surface (see the Supporting Information for experimetal details). The hexagonal protrusions represent the Moire pattern resulting from the lattice mismatch between the $\mathrm{ZnO}$ film and the surface. ${ }^{12,13}$ Figure $1 \mathrm{~b}$ illustrates the experiment, where TERS is recorded as a function of the relative displacement of the tip-sample distance $(\Delta z)$ under continuous-wave laser excitation with wavelength $\lambda_{\text {ext }}$. We used an electrochemically etched Ag tip further sharpened by focused ion beam (FIB) milling (Figure 1c and 1d), which enables control of localized surface plasmons in the STM junction. ${ }^{14}$ Figure le shows the TER spectra measured for 2-ML $\mathrm{ZnO}$ at different $\Delta z$ with $\lambda_{\text {ext }}=633$ $\mathrm{nm}$, which leads to tip-enhanced resonance Raman scattering where both electromagnetic (EM) and chemical enhancement (via electronic resonance) are operative simultaneously. ${ }^{15}$ During the measurement the bias voltage $\left(V_{\text {bias }}\right)$ is nominally set to zero, but there is an effective potential difference of $\sim 1$ $\mathrm{mV}$ due to an instrumental offset and a photovoltage, resulting in measurable currents in the junction. Figure if shows the tunneling current $\left(I_{\mathrm{t}}\right)$ versus $\Delta z . I_{\mathrm{t}}$ increases exponentially in the tunneling regime until it exhibits a jump-to-contact behavior, ${ }^{16}$ i.e., APC formation, which defines $\Delta z=0 \AA$. The conductance at $\Delta z=0 \AA$ is estimated to be $\sim 0.1 \mathrm{G}_{0}\left(=e^{2} /\right.$ $h=77.6 \mu \mathrm{S})$ by measuring the $I_{\mathrm{t}}-V_{\text {bias }}$ curve. In the tunneling regime $(\Delta z>0 \AA)$, the TERS intensity monotonically increases due to an increase of the plasmonic field in the junction. The intense peaks at $300-380 \mathrm{~cm}^{-1}$ can be assigned to the out-of-plane phonon modes of the $\mathrm{ZnO}$ film, whereas weak peaks at $200-300$ and $500-600 \mathrm{~cm}^{-1}$ are assigned to the in-plane modes. ${ }^{15}$ The surface selection rule for metals ${ }^{17}$ leads to much larger enhancement for the out-of-plane modes than the in-plane modes.

In the vicinity of the APC, several remarkable changes are observed (Figures $1 \mathrm{~g}$ and $1 \mathrm{~h}$ ). First, just before APC formation $(\Delta z=0.1 \AA)$, the relative intensity of the in-plane modes is significantly increased. Second, the overall TERS intensity suddenly increases at the APC. Third, a new characteristic peak at $\sim 400 \mathrm{~cm}^{-1}$ appears in the APC regime (denoted as "contact mode"). Forth, a continuous background suddenly appears upon APC formation. We found that the background intensity is correlated with the TERS intensity and it may be related to the plasmonic field in the junction (Figure S1). The Raman intensity shows a linear power dependence in both tunneling ${ }^{15}$ and APC regime (Figure S2).

We demonstrate that the APC can be formed and broken in a reversible manner. Figure 2 a displays a waterfall plot of the TER spectra during sequential approach and retraction of the $\mathrm{Ag}$ tip to and from 2-ML $\mathrm{ZnO}$ including both tunneling and APC regimes. The spectral evolution is symmetric with respect to the turning point $(\Delta z=-1.3 \AA$ ), and thus, the process is nondestructive (reversible). It is clear that the contact mode appears immediately at the APC and blue-shifts linearly (16.6 $\pm 1.0 \mathrm{~cm}^{-1} / \AA$ ) as $\Delta z$ is further reduced (the tip is "squeezed" to the $\mathrm{ZnO}$ film), indicating a variation in the bond strength. The TERS intensity decreases as the $\Delta z$ is further reduced in the APC regime $(\Delta z<0 \AA)$, which may result from the attenuation of the plasmonic field because the continuous 

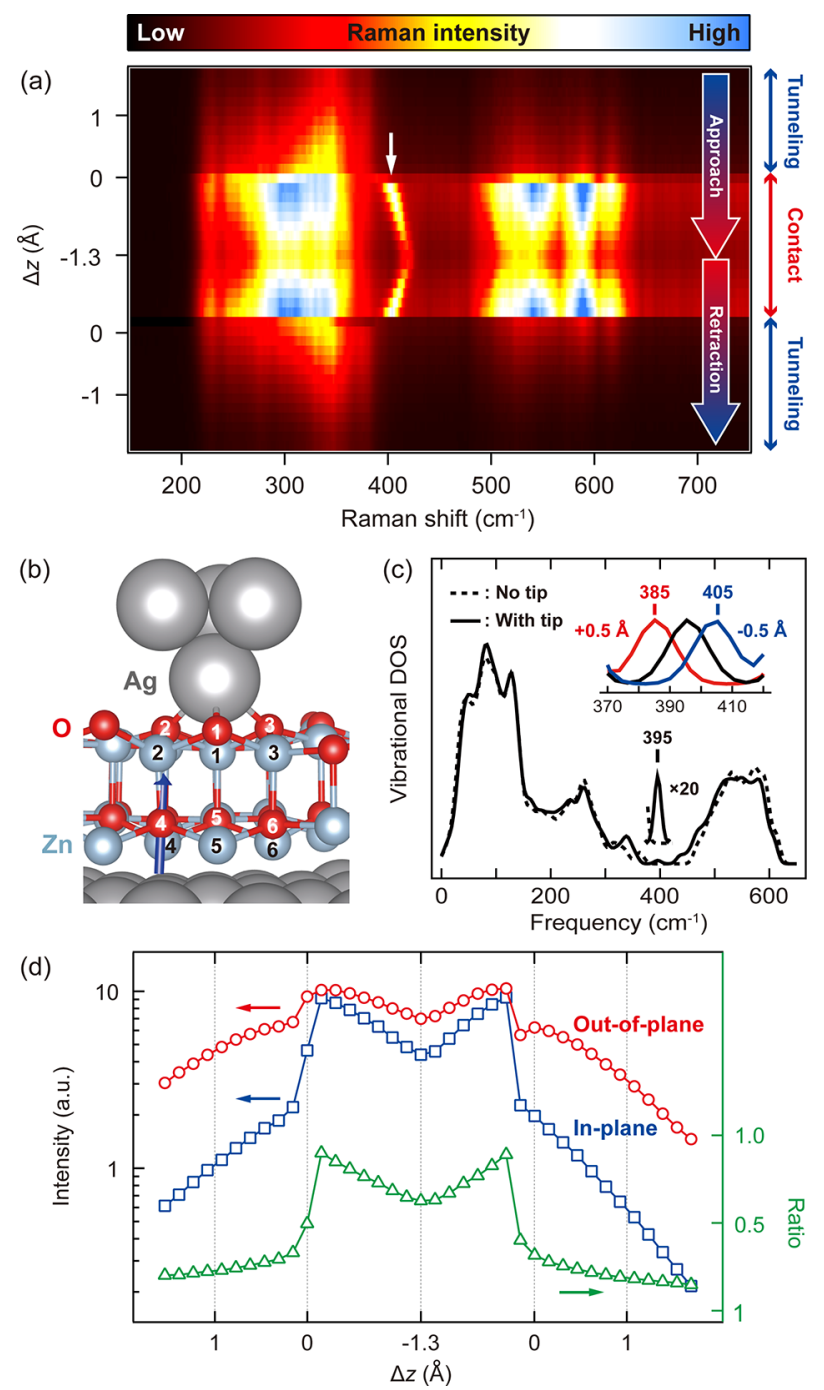

Figure 2. Evolution of TERS from tunneling to APC regime. (a) Waterfall plot of the gap-distance-dependent TER spectra recorded over 2-ML ZnO (Ag tip, $\lambda_{\text {ext }}=633 \mathrm{~nm}, F=0.36 \mathrm{~mW} \mu \mathrm{m}^{-2}$, $t_{\exp }=300$ $s$ per spectrum, $10 \mathrm{~K})$. The tip approaches and retracts toward and backward from the $\mathrm{ZnO}$ from the tunneling to the APC regime. (b) Optimized structure with the Ag tip. The characteristic vibrational mode resulting from the quantum point contact is shown by the blue arrow. (c) Calculated vibrational density of states (VDOS) with and without the Ag tip. The inset shows the corresponding VDOS as a function of tip displacement. (d) Integrated Raman intensity of the out-of-plane $\left(292-383 \mathrm{~cm}^{-1}\right)$ and in-plane $\left(489-644 \mathrm{~cm}^{-1}\right)$ modes as a function of $\Delta z$ (left axis). The continuous background in the TERS spectra is subtracted before integration. The ratio between these modes is also plotted (right axis).

background also decreases. We examined different Ag tips, and these observations were highly reproducible (Figure S3).

Our density functional theory (DFT) calculations (see the Supporting Information for details) reveal that the contact mode can be assigned to the local out-of-plane stretching mode of the $\mathrm{O}$ atom in the second layer (Figure $2 \mathrm{~b}$ ). When the APC is formed, the $\mathrm{Ag}$ tip interacts with the $\mathrm{O}$ atoms in the first layer. The resulting charge rearrangement in the fused junction (Figure S5) causes elongation of the in-plane $\mathrm{Zn}-\mathrm{O}$ bonds in both first and second layers underneath the tip, whereas the interlayer $\mathrm{Zn}-\mathrm{O}$ bond shrinks (the average bond length changes by $\sim 0.26 \AA$ ). As a consequence, the interlayer bond between $\mathrm{Zn}$ atom \#2 and $\mathrm{O}$ atom \#4 becomes stiffer, which leads to a new vibrational mode at $395 \mathrm{~cm}^{-1}$ (Figure 2c). In order to examine a "pushing" effect by the tip in the contact regime, we calculated the vibrational frequency of the new mode at different tip-surface distances and found that, indeed, the $\mathrm{Zn}-\mathrm{O}$ stretching mode blue-shifts at $20 \mathrm{~cm}^{-1} / \AA$ as the distance decreases because the bond gets stiffer (inset to Figure 2c). This is in excellent agreement with the experimental observation (Figure 2a), corroborating our assignment of the contact mode.

The relative intensity between the in- and out-of-plane phonon modes of 2-ML $\mathrm{ZnO}$ can be used to quantify the plasmonic field distribution as a function of $\Delta z$. The TERS intensities of these modes are primarily associated with the local field components perpendicular and parallel to the surface. The intensity of both modes exponentially varies in the tunneling regime (Figure $2 \mathrm{~d}$ ), and a moderate variation of their intensity ratio indicates the gradually increasing contribution of the in-plane field component and/or the field gradient of the tightly confined plasmon at the tip apex. ${ }^{18,19}$ The evolution of the plasmonic field in the STM junction is examined by electrodynamics simulations (Figure S6), which qualitatively reproduce a more rapid increase of the in-plane component, in good agreement with the experiment. On the other hand, when the APC is formed, the intensity of both in-plane and out-ofplane modes exhibit a discrete change and the relative intensity of the in-plane modes is largely increased. This should be associated with a sudden change of the polarizability of the $\mathrm{ZnO}$ film as discussed later. However, the plasmonic field distribution may not be affected upon APC formation because the overall spectral shape in TERS is essentially the same just before and after the contact except for the appearance of the contact mode (see the spectra at $\Delta z=0.1$ and $-0.3 \AA$ in Figure 1c). If the field distribution is significantly modified, selection rules for Raman scattering should be changed, and consequently, the TERS spectra would exhibit qualitative differences.

We now demonstrate that the dramatic enhancement of the TERS intensity upon APC formation is correlated with hybridization between the tip and the sample. In order to show a clear contrast to the case of the $\mathrm{ZnO}$ film, we examined a chemically inert $\mathrm{NaCl}$ film. Figures $3 \mathrm{a}$ and $3 \mathrm{~b}$ display the $I_{\mathrm{t}}-\Delta z$ curve and the waterfall plot of TER spectra, respectively, measured over 2-ML $\mathrm{NaCl}$ on the $\mathrm{Ag}(111)$ surface (Figure $3 c)$. The conductance at the APC is estimated to be $\sim 0.04 G_{0}$. Figure $3 \mathrm{~d}$ shows the TERS spectra at different $\Delta z$. The peak at $202 \mathrm{~cm}^{-1}$ is assigned to the transverse optical phonon mode of the $\mathrm{NaCl}$ film, ${ }^{20}$ whereas the peaks at lower frequencies $(<150$ $\mathrm{cm}^{-1}$ ) are assigned to the phonons of the Ag tip and/or substrate because they are also observed over the bare $\mathrm{Ag}(111)$ surface. Although the bulk $\mathrm{NaCl}$ is not Raman active, symmetry breaking occurs for ultrathin films on a metal surface, which is further lifted in the vicinity of the APC. As seen in Figure 3e, the TERS intensity rapidly increases in the tunneling regime. However, a sudden increase does not occur at the APC, although the continuous background pops up.

The continuous increase of the TERS intensity before APC formation can be attributed to EM enhancement arising from the confined plasmonic field, hence operative regardless of chemical properties of the sample. On the other hand, a sudden increase of the TERS intensity upon APC formation is critically affected by the chemical properties of the sample, whereby considerable enhancement is observed for the $\mathrm{ZnO}$ 


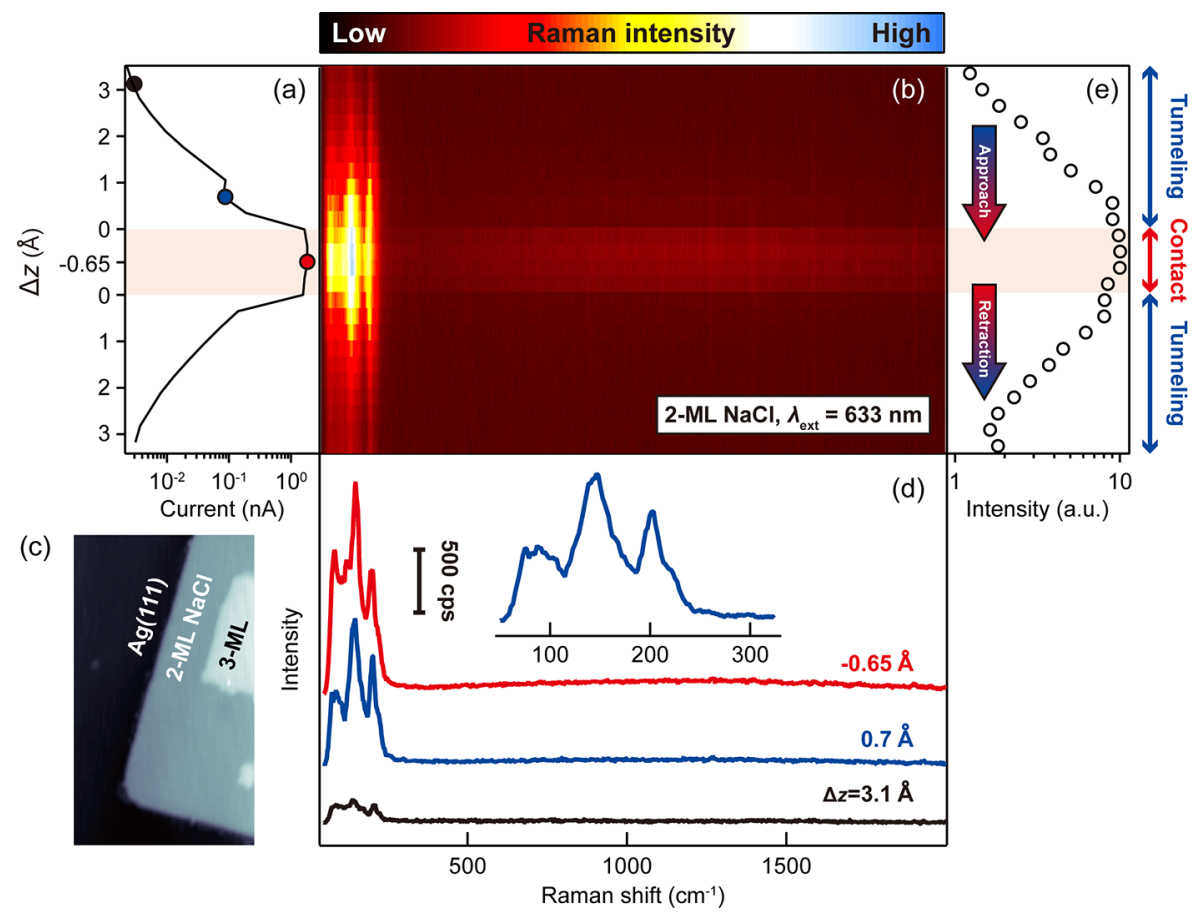

Figure 3. Gap-distance-dependent TERS measurement for 2-ML NaCl. (a) $I_{\mathrm{t}}$ versus $\Delta z$ acquired over 2-ML $\mathrm{NaCl}$ on $\mathrm{Ag}(111)$. The shaded area indicates the contact regime. $V_{\text {bias }}$ is set to $0 \mathrm{~V}$ nominally, but there is an offset bias of $\sim 1 \mathrm{mV}$ during the measurement. $\Delta z=0$ corresponds to the APC. The colored circles represent the point of the TERS spectra in (d). (b) Waterfall plot of the gap-distance-dependent TER spectra recoded over 2-ML NaCl (Ag tip, $\lambda_{\text {ext }}=633 \mathrm{~nm}, F=0.6 \mathrm{~mW} \mu \mathrm{m}^{-2}, t_{\exp }=5 \mathrm{~s}$ per spectrum, $10 \mathrm{~K}$ ). The tip first approaches toward the $\mathrm{NaCl}$ from the tunneling to contact regime. (c) STM image of $2-\mathrm{ML} \mathrm{NaCl}$ on $\mathrm{Ag}(111)\left(V_{\text {bias }}=1.0 \mathrm{~V}, I_{\mathrm{t}}=35 \mathrm{pA}, 45 \times 64 \mathrm{~nm}^{2}\right)$. (d) TER spectra at different distances. (e) TERS intensity of the peak at $\sim 202 \mathrm{~cm}^{-1}$.

film but not for the inert $\mathrm{NaCl}$ film. This remarkable difference may be associated with the charge transfer mechanism proposed by Persson ${ }^{21}$ which is further extended by Oren et $\mathrm{al}^{22}$ to nonzero temperature and current-carrying (fused) junctions. In the theoretical model, the enhancement of Raman scattering for a molecule adsorbed on a metal surface is attributed to charge transfer excitation between the sample (molecule) and the metal, thus the time-dependent polarization at the interface. This process is correlated with the hybridization between the sample and the metal which modifies the polarizability through formation of adsorptioninduced resonance states. In electrically fused junctions, the sample can simultaneously hybridize with metals at both sides (surface and tip in the STM configuration), making charge transfer enhancement operative through the metal-samplemetal system. Intuitively, in an electrically fused junction, more electrons can contribute to the process compared to the sample-metal system, thus increasing the polarizability of the sample. The theoretical model reveals that the Raman scattering function (defined in eq 14 in ref 21) becomes about twice as large in an electrically fused metal-samplemetal junction than that for the sample-metal system, ${ }^{22}$ which eventually leads to a 4-fold larger enhancement in the Raman intensity (proportional to the square of the scattering function). This additional enhancement in the fused junction is consistent with the change in the TERS intensity upon APC formation observed for the $\mathrm{ZnO}$ film. However, the hybridization is almost negligible for the chemically inert $\mathrm{NaCl}$ film, which is supported, for instance, by the fact that pristine molecular orbitals can be observed for molecules adsorbed there. ${ }^{23}$ The lack of hybridization results in no additional charge transfer enhancement upon APC formation. We found that the charge transfer mechanism leads to a significant enhancement even for off-resonance $\lambda_{\text {ext }}$ at which TERS is very weak (or unobservable) in the tunneling regime (Figure S7).

Finally, we would like to note that thermal instability of plasmonic junctions has a critical impact on the TERS enhancement in the vicinity of the APC. As shown in our previous TERS experiments on the $\mathrm{ZnO}$ films at $80 \mathrm{~K}$, ${ }^{15}$ neither APC formation nor sudden enhancement was observed at vanishing gap distances. Instead, significant attenuation of the TERS intensity routinely takes place. This observation suggests that the absence of the APC formation at $80 \mathrm{~K}$ does not result in the charge transfer enhancement and that the reduction of the plasmonic field by electron tunneling ${ }^{24}$ is more pronounced. Atomic-scale structural relaxation (rearrangement) is expected to be crucial in light scattering in the vicinity of the APC because the plasmonic properties in ångström-scale cavities are extremely sensitive to subtle geometrical changes. A critical impact of thermal fluctuations was observed for ultrasensitive SERS in plasmonic picocavities, where diffusion of $\mathrm{Au}$ atoms creates and annihilates atomistic hot spots. ${ }^{25}$ Therefore, in-depth understanding of thermally/ optically induced atomic-scale structural fluctuations (instability) as well as light-matter interactions in ångström-scale the near-field optical spectroscopy for "realistic" systems, e.g., under ambient conditions and in solutions.

As a perspective, the strong enhancement at APCs will be applicable to other materials to increase the sensitivity, which can expand the capability of TERS. In addition, our approach can be extended to study single-molecule junctions. In combination with inelastic electron tunneling spectroscopy, we can provide detailed information not only on junction structures but also on chemical bond formation, electron- 
phonon coupling, local heating, and optoelectronic properties. Moreover, TERS in atomic-scale cavities is intimately related to fundamental questions in quantum plasmonics, ${ }^{26,27}$ and our approach will also provide microscopic insights into intriguing atomic-scale light-matter interactions. ${ }^{28}$

\section{ASSOCIATED CONTENT}

\section{SI Supporting Information}

The Supporting Information is available free of charge at https://pubs.acs.org/doi/10.1021/acs.nanolett.0c01791.

Details of experiments, correlation between the TERS intensity and the continuous background in the APC regime, incident laser power dependence of the TERS intensity in the APC regime, TERS spectra of the $\mathrm{Ag}$ tip- $\mathrm{ZnO}$ junction measured by different tips, details of DFT calculations, stable structure of the $\mathrm{Ag}$ tip- $\mathrm{ZnO}$ junction, calculated charge density difference upon formation of the APC, electrodynamic simulations of the near-field distribution in the STM junction, and wavelength dependence of TERS in transition from the tunneling to the APC regime (PDF)

\section{AUTHOR INFORMATION}

\section{Corresponding Author}

Takashi Kumagai - Department of Physical Chemistry, FritzHaber Institute of the Max-Planck Society, 14195 Berlin, Germany; JST-PRESTO, Kawaguchi, Saitama 332-0012, Japan; Center for Mesoscopic Sciences, Institute for Molecular Science, Okazaki 444-8585, Japan; 이이.org/0000-00017029-062X; Email: kuma@fhi-berlin.mpg.de, kuma@ ims.ac.jp

\section{Authors}

Shuyi Liu - Department of Physical Chemistry, Fritz-Haber Institute of the Max-Planck Society, 14195 Berlin, Germany

Borja Cirera - Department of Physical Chemistry, Fritz-Haber Institute of the Max-Planck Society, 14195 Berlin, Germany

Yang Sun - Global Research Center for Environment and Energy based on Nanomaterials Science, National Institute for Materials Science, Tsukuba, Ibaraki 305-0044, Japan

Ikutaro Hamada - Global Research Center for Environment and Energy based on Nanomaterials Science, National Institute for Materials Science, Tsukuba, Ibaraki 305-0044, Japan; Department of Precision Science and Technology, Graduate School of Engineering, Osaka University, Suita, Osaka 5650871, Japan; 이이. orcid.org/0000-0001-5112-2452

Melanie Müller - Department of Physical Chemistry, FritzHaber Institute of the Max-Planck Society, 14195 Berlin,

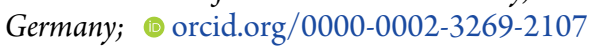

Adnan Hammud - Department of Inorganic Chemistry, FritzHaber Institute of the Max-Planck Society, 14195 Berlin, Germany

Martin Wolf - Department of Physical Chemistry, Fritz-Haber Institute of the Max-Planck Society, 14195 Berlin, Germany

Complete contact information is available at:

https://pubs.acs.org/10.1021/acs.nanolett.0c01791

\section{Notes}

The authors declare no competing financial interest.

\section{ACKNOWLEDGMENTS}

The authors thank Chenfang Lin, Markus Raschke, Robert Schlögl, Mariana Rossi, and Leonhard Grill for careful reading of the manuscript and providing valuable comments. T.K. acknowledges the support by JST-PRESTO (JPMJPR16S6). T.K. and M.W. acknowledge the support by the Deutsche Forschungsgemeinschaft through Sfb951. The numerical calculations were performed using the Numerical Materials Simulator at the National Institute for Materials Science.

\section{REFERENCES}

(1) Zrimsek, A. B.; Chiang, N.; Mattei, M.; Zaleski, S.; McAnally, M. O.; Chapman, C. T.; Henry, A.-I.; Schatz, G. C.; Van Duyne, R. P. Single-Molecule Chemistry with Surface- and Tip-Enhanced Raman Spectroscopy. Chem. Rev. 2017, 117, 7583-7613.

(2) Benz, F.; Schmidt, M. K.; Dreismann, A.; Chikkaraddy, R.; Zhang, Y.; Demetriadou, A.; Carnegie, C.; Ohadi, H.; de Nijs, B.; Esteban, R.; Aizpurua, J.; Baumberg, J. J. Single-molecule optomechanics in "picocavities. Science 2016, 354, 726-729.

(3) Barbry, M.; Koval, P.; Marchesin, F.; Esteban, R.; Borisov, A. G.; Aizpurua, J.; Sánchez-Portal, D. Atomistic near-field nanoplasmonics: Reaching atomic-scale resolution in nanooptics. Nano Lett. 2015, 15, 3410-3419.

(4) Urbieta, M.; Barbry, M.; Zhang, Y.; Koval, P.; Sánchez-Portal, D.; Zabala, N.; Aizpurua, J. Atomic-Scale Lightning Rod Effect in Plasmonic Picocavities: a Classical View to a Quantum Effect. ACS Nano 2018, 12, 585-595.

(5) Kern, J.; Großmann, S.; Tarakina, N. V.; Häckel, T.; Emmerling, M.; Kamp, M.; Huang, J.-S.; Biagioni, P.; Prangsma, J. C.; Hecht, B. Atomic-Scale Confinement of Resonant Optical Fields. Nano Lett. 2012, 12, 5504-5509.

(6) Lee, J.; Crampton, K. T.; Tallarida, N.; Apkarian, V. A. Visualizing vibrational normal modes of a single molecule with atomically confined light. Nature 2019, 568, 78-82.

(7) Zhang, Y.; Yang, B.; Ghafoor, A.; Zhang, Y.; Zhang, Y.-F.; Wang, R.-P.; Yang, J.-L.; Luo, Y.; Dong, Z.-C.; Hou, J. G. Visually Constructing the Chemical Structure of a Single Molecule by Scanning Raman Picoscopy. Natl. Sci. Rev. 2019, 6, 1169.

(8) Savage, K. J.; et al.et al Revealing the quantum regime in tunnelling plasmonics. Nature 2012, 491, 574-577.

(9) Banik, M.; Apkarian, V. A.; Park, T. H.; Galperin, M. Raman Staircase in Charge Transfer SERS at the Junction of Fusing Nanospheres. J. Phys. Chem. Lett. 2013, 4, 88-92.

(10) El-Khoury, P. Z.; Hu, D.; Apkarian, V. A.; Hess, W. P. Raman Scattering at Plasmonic Junctions Shorted by Conductive Molecular Bridges. Nano Lett. 2013, 13, 1858-1861.

(11) Banik, M.; El-Khoury, P. Z.; Nag, A.; Rodriguez-Perez, A.; Guarrottxena, N.; Bazan, G. C.; Apkarian, V. A. Surface-Enhanced Raman Trajectories on a Nano-Dumbbell: Transition from Field to Charge Transfer Plasmons as the Spheres Fuse. ACS Nano 2012, 6 (11), 10343-10354.

(12) Kumagai, T.; Liu, S.; Shiotari, A.; Baugh, D.; Shaikhutdinov, S.; Wolf, M. Local electronic structure, work function, and line defect dynamics of ultrathin epitaxial $\mathrm{ZnO}$ layers on a $\mathrm{Ag}(111)$ surface. J. Phys.: Condens. Matter 2016, 28, 494003.

(13) Liu, S.; Shiotari, A.; Baugh, D.; Wolf, M.; Kumagai, T. Enhanced resolution imaging of ultrathin $\mathrm{ZnO}$ layers on $\mathrm{Ag}(111)$ by multiple hydrogen molecules in a scanning tunneling microscope junction. Phys. Rev. B: Condens. Matter Mater. Phys. 2018, 97, 195417.

(14) Böckmann, H.; Liu, S.; Müller, M.; Hammud, A.; Wolf, M.; Kumagai, T. Near-Field Manipulation in a Scanning Tunneling Microscope Junction with Plasmonic Fabry-Pérot Tips. Nano Lett. 2019, 19, 3597-3602.

(15) Liu, S.; Müller, M.; Sun, Y.; Hamada, I.; Hammud, A.; Wolf, M.; Kumagai, T. Resolving the Correlation between Tip-Enhanced Resonance RamanScattering and Local Electronic States with $1 \mathrm{~nm}$ Resolution. Nano Lett. 2019, 19, 5725-5731. 
(16) Kröger, J.; Ńeel, N.; Limot, L. Contact to single atoms and molecules with the tipof a scanning tunnelling microscope. J. Phys.: Condens. Matter 2008, 20, 223001.

(17) Moskovits, M.; Suh, J. S. Surface selection rules for surfaceenhanced Raman spectroscopy: calculations and application to the surface-enhanced Raman spectrum of phthalazine on silver. J. Phys. Chem. 1984, 88, 5526-5530.

(18) Meng, L.; Yang, Z.; Chen, J.; Sun, M. Effect of Electric Field Gradient on Sub-nanometer Spatial Resolution of Tip-enhanced Raman Spectroscopy. Sci. Rep. 2015, 5, 9240.

(19) Chen, X.; Jensen, L. Morphology dependent near-field response in atomistic plasmonic nanocavities. Nanoscale 2018, 10, 1141011417.

(20) Schmunk, R. E.; Winder, D. R. Lattice dynamics of sodium chloride at room temperature. J. Phys. Chem. Solids 1970, 31, 131141.

(21) Persson, B. N. J. On the theory of surface-enhanced Raman scattering. Chem. Phys. Lett. 1981, 82, 561-565.

(22) Oren, M.; Galperin, M.; Nitzan, A. Raman scattering from molecular conduction junctions: Charge transfer mechanism. Phys. Rev. B: Condens. Matter Mater. Phys. 2012, 85, 115435.

(23) Repp, J.; Meyer, G.; Stojković, S. M.; Gourdon, A.; Joachim, C. Molecules on Insulating Films: Scanning-Tunneling Microscopy Imaging of Individual Molecular Orbitals. Phys. Rev. Lett. 2005, 94, No. 026803.

(24) Zuloaga, J.; Prodan, E.; Nordlander, P. Quantum Description of the Plasmon Resonances of a Nanoparticle Dimer. Nano Lett. 2009, 9, 887-891.

(25) Carnegie, C.; Griffiths, J.; de Nijs, B.; Readman, C.; Chikkaraddy, R.; Deacon, W. M.; Zhang, Y.; Szabó, I.; Rosta, E.; Aizpurua, J.; Baumberg, J. J. Room-Temperature Optical Picocavities below $1 \mathrm{~nm}^{3}$ Accessing Single-Atom Geometries. J. Phys. Chem. Lett. 2018, 9, 7146-7151.

(26) Tame, M. S.; McEnery, K. R.; Özdemir, Ş. K.; Lee, J.; Maier, S. A.; Kim, M. S. Quantum plasmonics. Nat. Phys. 2013, 9, 329-340.

(27) Zhu, W.; Esteban, R.; Borisov, A. G.; Baumberg, J. J.; Nordlander, P.; Lezec, H. J.; Aizpurua, J.; Crozier, K. B. Quantum mechanical effects in plasmonic structures with subnanometre gaps. Nat. Commun. 2016, 7, 11495.

(28) Baumberg, J. J.; Aizpurua, J.; Mikkelsen, M. H.; Smith, D. R. Extreme nanophotonics from ultrathin metallic gaps. Nat. Mater. 2019, 18, 668-678. 